\section{Literatur}

Daft, $R$. L., Lengel, $R$. H., Information richness: A new approach to managerial behavior and organizational design, in: Research in Organizational Behavior, Vol. 6 (1984), 191-233.

Emrich, C., Multi-Channel-Communications- und Marketing-Management, Wiesbaden 2008.

Fisher, R., Patton, B. M., Ury, W., Das Harvard-Konzept. Der Klassiker der Verhandlungstechnik, 22. Aufl., Frankfurt/Main u.a. 2004.

Khabyuk, 0., Kommunikationsmodelle. Grundlagen - Anwendungsfelder Grenzen, Stuttgart 2019.

Lasswell, H. D., The structure and function of communication in society, in: L. Bryson (Hrsg.), The communication of ideas. A series of addresses, New York 1964, S. 37-51.

Lazarsfeld, P. F., Berelson, B., Gaudet, H., The People's Choice. How the voter makes up his mind in a presidential campaign, 3. Aufl., New York/London 1968.

Maletzke, G., Psychologie der Massenkommunikation. Theorie und Systematik, Hamburg 1963.

Six, U., Gleich, U., Gimmler, R. (Hrsg.), Kommunikationspsychologie - Medienpsychologie, Weinheim 2007.

Watzlawick, P., Beavin, J. B., Jackson, D. D., Menschliche Kommunikation. Formen, Störungen, Paradoxien, 12. Aufl., Bern 2011.

\title{
Verleihung des deutschen Studienpreises 2020
}

Die Körber Stiftung verleiht auch in diesem Jahr den deutschen Studienpreis für exzellente Dissertationen aus den Fachbereichen der Sozialwissenschaften, Natur- und Technikwissenschaften sowie Geistes- und Kulturwissenschaften. Mit dem Preis werden Wissenschaftlerinnen und Wissenschaftler ausgezeichnet, die im Rahmen ihrer Promotion zu gesellschaftlich relevanten Themen geforscht und anwendungsbezogene Ergebnisse hervorgebracht haben. Die gesellschaftliche Bedeutung der Ergebnisse ist das ausschlaggebende Kriterium.

Insgesamt verleiht die Körber Stiftung, die unter der Schirmherrschaft des Bundestagspräsidenten, Dr. Wolfgang Schäuble, steht, Preise im Wert von $100.000 €$, wobei die drei ersten Preise mit jeweils $25.000 €$ dotiert sind. Akademiker sollen durch den Preis dazu ermutigt werden, den gesellschaftlichen Wert ihrer wissenschaftlichen Arbeit herauszustellen und im Rahmen einer öffentlichen Debatte zu verteidigen. Neben fachlicher Expertise sollten Bewerber ebenfalls kommunikative Qualitäten aufweisen, um, im Zuge einer Auswahltagung, die Jury von der Relevanz ihrer vorliegenden Ergebnisse zu überzeugen.

Es können alle Promovierten teilnehmen, die im Jahr 2020 ihre Promotion mit magna oder summa cum laude abgeschlossen haben. Der Einsendeschluss ist der 01.03.2021. Weitere Informationen finden Sie unter www.studienpreis. de. 